\title{
Artigo/Article
}

\section{Perfil epidemiológico da hanseníase em um município brasileiro, no período de 2000 a 2006}

\author{
Epidemiological profile of leprosy in a Brazilian municipality between 2000 and 2006
}

Sybelle de Souza Castro Miranzi ${ }^{1}$, Lívia Helena de Morais Pereira ${ }^{2}$ e Altacílio Aparecido Nunes ${ }^{3}$

\begin{abstract}
RESUMO
Introdução: A hanseníase é considerada um grande problema de saúde pública nos países em desenvolvimento. Estima-se que somente $1 / 3$ dos doentes sejam notificados e que, dentre esses, muitos fazem tratamento irregular ou o abandonam, aumentando o impacto da doença. Assim o objetivo desse artigo foi descrever o perfil epidemiológico da população com diagnóstico de hanseníase, no município de Uberaba, Estado de Minas Gerais, Brasil, no período de 2000 a 2006. Métodos: Trata-se de um estudo retrospectivo, que utilizou os dados secundários de notificação de casos hanseníase do Sistema de Informação de Agravos de Notificação do Ministério da Saúde do Brasil. Resultados: Foram registrados 455 casos da doença, sendo 55,4\% do sexo masculino, a faixa etária dos 34 a 49 anos $(31,4 \%)$ foi a mais afetada, houve registro de nove $(2 \%)$ casos de hanseníase em menores de 15 anos. A forma clínica prevalente foi a dimorfa $(69,1 \%)$ e a classe operacional foi a multibacilar (87\%). Tais achados são preocupantes, considerando-se que são de faixa etária economicamente ativa e potencialmente, os principais disseminadores da doença. Conclusões: O relato de que a maioria dos casos eram multibacilares, indica diagnósticos tardios, assim, torna-se necessário descentralizar o serviço de hanseníase e capacitar mais profissionais para possibilitar diagnóstico e tratamentos mais precoces.
\end{abstract}

Palavras-chaves: Hanseníase. Epidemiologia. Prevalência. Incidência.

\begin{abstract}
Introduction: Leprosy is considered to be a major public health problem in developing countries. It is estimated that notifications are issued only in relation to $1 / 3$ of the patients and that, among these patients, many undergo irregular treatment or drop out, thus increasing the impact of the disease. The objective this paper was to describe the epidemiological profile of the population with a diagnosis of leprosy in the municipality of Uberaba, State of Minas Gerais, Brazil, between 2000 and 2006. Methods: This was a retrospective study using data from notifications of leprosy cases in the Notifiable Disease Information System of the Brazilian Ministry of Health. Results: There were 455 recorded cases of leprosy, and 55.4\% of these were among males. The most affected age group was 34 to 49 years (31.4\%), while nine cases of leprosy $(2 \%)$ were found in children under the age of 15 years. The commonest clinical form was the borderline type $(69.1 \%)$ and the operational class was multibacillary (87\%). These findings are a matter of concern, considering that the age group that is most economically active is potentially the principal group spreading the disease. Conclusions: The finding that $87 \%$ of the cases were multibacillary indicates that the diagnoses were late. It is therefore necessary to decentralize the leprosy care services and train more professionals to enable earlier diagnosis and treatment.
\end{abstract}

Key-words: Leprosy. Epidemiology. Prevalence. Incidence.

1. Departamento de Medicina Social, Universidade Federal do Triângulo Mineiro, Uberaba, MG. 2. Pós-Graduação, Universidade Federal do Triângulo Mineiro, Uberaba, MG. 3. Departamento de Medicina Social, Faculdade de Medicina de Ribeirão Preto, Universidade de São Paulo, Ribeirão Preto, SP.

Endereço para correspondência: Dra. Sybelle de Souza Castro Miranzi. Dept ${ }^{\circ}$ de Medicina Social/UFTM. Rua Constituição 920, Bairro Abadia, 38026-280 Uberaba, MG

Tel: $55343318-5572$

e-mail: sybelle@mednet.com.br

Recebido para publicação em 27/05/2009

Aceito em 22/12/2009

\section{INTRODUÇÃO}

A hanseníase é considerada um grande problema de saúde pública nos países em desenvolvimento ${ }^{1}$.Em 1991, a Assembléia Mundial de Saúde determinou que a prevalência de hanseníase em países endêmicos chegasse a menos de 1/10.000 até o ano de $2000^{2}$. Obteve-se uma redução da prevalência em aproximadamente $85 \%$ dos casos em todo mundo, porém não alcançou a meta inicial proposta ${ }^{3,4}$. Com o intuito de reduzir ainda mais os valores de detecção e prevalência da doença e reparar as questões não resolvidas no Plano de Eliminação a Organização Mundial de Saúde (OMS) foi lançado A Estratégia Global para Maior Redução da Carga da Hanseníase e a Sustentação das Atividades de Controle da Hanseníase proposta para 2006-2010. Os resultados esperados ao fim de 2010 são a diminuição dos níveis da hanseníase para valores muito baixos; uma elevação da qualidade dos serviços de saúde com profissionais mais capacitados para detecção da doença, melhor sistema de gestão, melhora da qualidade dos registros dos casos e acessibilidade a serviços especializados; recursos para garantir a prevenção de incapacidades e a reabilitação quando necessárias e promover a integração e parcerias com outras instituições.

Atualmente, as taxas de hanseníase mundial reduziram em aproximadamente $90 \%$ quando comparadas há duas décadas atrás, observando-se uma queda de $37,8 \%$ na incidência da doença no Brasil, entre 1998 e $2003^{4}$. Entretanto, devido ao esforço do Ministério da Saúde, em dois anos houve uma diminuição em $24,3 \%$ dos casos de hanseníase e em 2005 a taxa de incidência foi de 2,1 casos em 10.000. Isso, em parte, deve-se ao aumento de $41,1 \%$ dos serviços de diagnóstico e tratamento no sistema público de saúde ${ }^{5}$.

A história da hanseníase no Brasil mostra que a prevalência da doença teve uma redução importante, ainda que a taxa de detecção não tenha diminuído efetivamente, apresentando uma elevação do ano de 
2005 para 2006. O quadro deve-se à endemia oculta (pois é insipiente a busca ativa dos doentes nas áreas endêmicas), aos diagnósticos tardios, deficiência nos programas público-assistenciais, precariedade dos serviços de saúde, abandono do tratamento, baixo nível de esclarecimento da população, ao preconceito e estigma que recaem sobre a doença ${ }^{1,6,7}$. Estima-se que somente $1 / 3$ dos portadores do bacilo de Hansen esteja notificado e que, dentre esses, muitos fazem um tratamento irregular ou o abandonam, tendo como conseqüência bacilos resistentes às medicações e que podem levar a dificuldades no tratamento da doença, aumentando o problema nacional da hanseníase ${ }^{7,8}$.

Apesar da eficiência em reduzir casos de hanseníase, os planos de eliminação não foram suficientes para desclassificar a doença como problema de Saúde Pública. O Plano de Eliminação Nacional de Hanseníase em âmbito municipal (2006-2010) tem a meta de eliminação da doença até 2010 com ações como a redução da prevalência para valores abaixo de $1 / 10.000$, monitorização dos registros de casos novos, o fortalecimento da vigilância epidemiológica, capacitação dos profissionais de saúde e uma rede eficiente de referência e contra-referência ${ }^{5}$.

O Estado de Minas Gerais apresenta um quadro melhor comparado ao Brasil. A taxa de incidência foi de 1,5 casos por 10 mil habitantes no ano de 2005. Esse valor decai em 2006 e chega a $1,2 / 10.000$. Um dado interessante é que Minas Gerais é o primeiro estado a ter uma legislação específica para a doença, a Política de Educação Preventiva Contra a Hanseníase e de Combate ao Preconceito, sua questão principal é desestigmatizar a doença, visto que ela possui cura e se diagnosticada precocemente, evita sequelas?.

Apesar dos bons índices verificados em Minas Gerais, não significa que a doença será erradicada por completo e ações de combate à eliminação da hanseníase devem continuar intensificadas nos municípios mais atingidos no estado como Uberaba, Uberlândia, Paracatu, Governador Valadares, Ituiutaba ${ }^{10}$. Uberaba apresenta uma queda dos níveis de prevalência, entretanto, seus valores ainda ultrapassam os de Minas Gerais, que em 2006, teve uma taxa de 1,02 por 10.000 habitantes, que a classifica como de alto nível endêmico e de média magnitude, em contrapartida o estado apresentava uma taxa de $0,83^{6}$.

Para contribuir com a monitorização da doença e com o fortalecimento da vigilância epidemiológica considerou-se importante descrever o perfil epidemiológico da hanseníase em Uberaba/MG, com o intuito caracterizar a tendência e a magnitude da doença no município.

\section{MÉTODOS}

Este foi um estudo epidemiológico observacional, retrospectivo, que utilizou o sistema informatizado de dados das notificações de hanseníase, vinculado à Secretaria Municipal de Saúde e ao DATASUS abrangendo o período entre 2000 e 2006. Esse banco de dados é constituído por todos os casos de hanseníase notificados e confirmados em residentes de Uberaba, através da Ficha Individual de Notificação/Investigação de Hanseníase, arquivada no Sistema de Informação de Agravos de Notificação (SINAN). As variáveis do estudo foram divididas em sete categorias de análise: caracterização do caso (sociodemográficos); antecedentes epidemiológicos, dados clínicos; de atendimento, laboratoriais, de tratamento e medidas de controle. Os indicadores utilizados para o estudo foram os coeficientes de incidência e de prevalência por 100.000 ou 10.000 habitantes, respectivamente.

\section{Critérios de inclusão e exclusão}

Foram incluídos no estudo todos os casos notificados e com diagnóstico confirmado de hanseníase. Contudo, foram excluídos da análise todos os casos que, apesar de notificados, não apresentavam confirmação diagnóstica ou que continha inconsistências.

\section{Análise estatística}

Foram realizadas análises exploratórias (descritivas) dos dados, a partir da apuração de frequências simples absolutas e percentuais para as variáveis categóricas e organização dos resultados em tabelas e gráficos. Para comparação de diferenças e distribuição entre proporções, foram empregados os testes qui-quadrado e $\mathrm{Z}$. Para verificação de associação entre variáveis, empregou-se como estimador de magnitude o odds ratio (OR) e seu intervalo de confiança a 95\% (IC95\%). O nível de significância adotado em todas as análises foi de $5 \%(\mathrm{p}<0,05)$. Os dados foram gerenciados no software Epi-info 2000 e analisados no SPSS versão 10.0.

\section{Ética}

O Projeto de Pesquisa foi aprovado pelo Comitê de Ética em Pesquisa da Universidade Federal do Triângulo Mineiro.

\section{RESULTADOS}

Foram registrados 455 casos de hanseníase no período entre 2000 e 2006, pela Secretaria Municipal de Saúde de Uberaba, com uma média de 65 casos novos por ano, com média anual de taxa de incidência de 27 casos/100.000 habitantes (Figura 1). Sendo que em 2004, ano com o maior número de notificações $(20,5 \%$ do total), registrou-se 93 casos com coeficiente de incidência de 32,2 casos/100.000 habitantes.

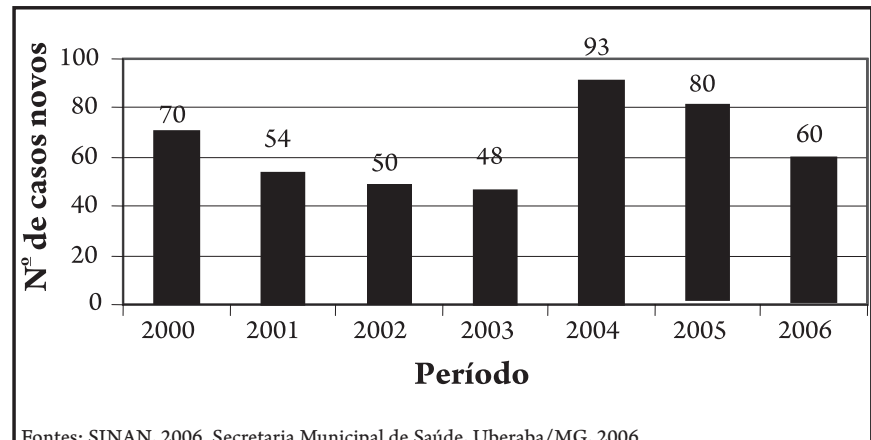

FIGURA 1 - Distribuição dos casos de hanseníase, diagnosticados no período de 2000 a 2006, no município de Uberaba/MG.

A detecção, entre os anos de 2000 e 2006, foi feita em 55,6\% dos casos, pela forma de encaminhamento e $35,3 \%$ por demanda espontânea. A porcentagem de pacientes que obtiveram cura foi de $83,9 \%$. A distribuição dos casos por gênero revelou que $252(55,4 \%)$ portadores da doença eram homens ( $\mathrm{p}<0,05)$. Quanto ao local de moradia, 82,8\% moravam na zona urbana $(\mathrm{p}<0,05)$. Na avaliação da raça/cor, os pardos foram a maioria, com $33,4 \%$ dos casos, seguidos pelos brancos com $29,8 \%(p=0,37)$, com a raça negraapresentandoamenorproporçãodecasosquandocomparadaàsoutras $(p<0,05)$. A forma clínica prevalente em todos os anos analisados foi 
a tipo dimorfa $(69,1 \%)$, seguida do tipo virchowiana $(17,1 \%)$, que comparadas com as formas indeterminadas e tuberculóide, apresentaram diferença significativa $(p<0,05)$. A forma multibacilar foi a predominante $\mathrm{e}$, em relação à paucibacilar, apresentou diferença $(\mathrm{p}<0,05)$ importante (Tabela 1).

Com relação à distribuição etária (Tabela 2), as faixas de 5 a 9 anos e 50 a 64 anos apresentaram distribuição igual entre os sexos, enquanto dos 15 aos 19 anos predominou o sexo feminino com $72,7 \%$ dos casos $(p<0,05)$. Nas demais faixas etárias, o sexo masculino teve uma maior porcentagem, sendo que na análise geral o sexo masculino representou 55,4\% do total $(\mathrm{p}<0,05)$. Com relação aos menores de 15 anos foram notificados nove casos de hanseníase, seis desses eram dimorfos, dois indeterminados e um tuberculóide.

A maioria dos portadores de hanseníase estudou em média de quatro a sete anos (34,9\%). A faixa de 20 a 34 anos foi a que

TABELA 1 - Distribuição dos registros, segundo sexo, zona de residência, raça/ cor, classificação operacional atual, forma clínica, modo de detecção e tipo de alta entre os casos de hanseníase, Uberaba-MG, 2000 a 2006.

\begin{tabular}{|c|c|c|c|}
\hline Características & Número & Percentagem & $\mathbf{p}$ \\
\hline \multicolumn{4}{|l|}{ Sexo } \\
\hline masculino & 252 & 55,4 & $<0,05$ \\
\hline feminino & 203 & 44,6 & \\
\hline total & 455 & 100,0 & \\
\hline \multicolumn{4}{|l|}{ Zona de residência } \\
\hline urbana & 377 & $82,8^{*}$ & $<0,05^{*}$ \\
\hline rural & 6 & $1,3^{*}$ & \\
\hline ignorada/branco & 72 & 15,8 & \\
\hline total & 455 & 100,0 & \\
\hline \multicolumn{4}{|l|}{ Raça/cor } \\
\hline ignorada/branca & 128 & 28,1 & $<0,05 @$ \\
\hline branca & 136 & $29,8 \#$ & \\
\hline preta & 39 & 8,5 & \\
\hline parda & 152 & 33,4 \# & $0,37 \#$ \\
\hline total & 455 & 100,0 & \\
\hline \multicolumn{4}{|c|}{ Classificação operacional atual } \\
\hline paucibacilar & 59 & 12,9 & $<0,05$ \\
\hline multibacilar & 396 & 87,1 & \\
\hline total & 455 & 100,0 & \\
\hline \multicolumn{4}{|l|}{ Forma clínica } \\
\hline indeterminada & 24 & 5,3 & $<0,05 ¥$ \\
\hline tuberculóide & 36 & 7,9 & \\
\hline dimorfa & 317 & 69,7 & \\
\hline virchowiana & 78 & 17,1 & $0,91^{9}$ \\
\hline total & 455 & 100,0 & \\
\hline \multicolumn{4}{|l|}{ Modo de detecção } \\
\hline encaminhamento & 253 & 55,6 & $<0,05$ \\
\hline demanda espontânea & 161 & 35,3 & \\
\hline exame coletividade & 2 & 0,4 & \\
\hline total & 416 & 91,3 & \\
\hline \multicolumn{4}{|l|}{ Tipo de alta } \\
\hline cura & 382 & 83,9 & $<0,05$ \\
\hline erro diagnóstico & 1 & 0,2 & \\
\hline total & 383 & 84,1 & \\
\hline
\end{tabular}

* comparação entre população urbana e rural, @ comparação entre todas as raças, \# comparação entre raça branca e parda, ¥ comparação entre todas as formas clínicas, $\mathbf{I}$ comparação entre formas clínicas indeterminada e tuberculóide
TABELA 2 - Distribuição dos percentuais quanto à classificação operacional atual versus sexo, faixa etária e escolaridade em portadores de hanseníase Uberaba/MG, 2000 a 2006.

\begin{tabular}{|c|c|c|c|c|c|c|}
\hline \multirow[b]{2}{*}{ Características } & \multicolumn{2}{|c|}{ Feminino } & \multicolumn{2}{|c|}{ Masculino } & \multirow[b]{2}{*}{ OR (IC95\%) } & \multirow[b]{2}{*}{$p$} \\
\hline & $\mathrm{n}^{\circ}$ & $\%$ & $\mathrm{n}^{\mathrm{o}}$ & $\%$ & & \\
\hline \multicolumn{7}{|c|}{ Classificação atual X sexo } \\
\hline paucibacilar & 12 & 6,0 & 55 & 21,7 & $4,4(2,2-9,0)^{*}$ & $<0,05$ \\
\hline multibacilar & 191 & 94,0 & 197 & 78,3 & & \\
\hline
\end{tabular}

\section{Faixa etária (ano) X sexo}

$\begin{array}{lrrrr}<1 & 1 & 100,0 & 0 & - \\ 5 \text { a } 9 & 2 & 50,0 & 2 & 50,0 \\ 10 \text { a } 14 & 1 & 25,0 & 3 & 75,0 \\ 15 \text { a } 19 & 8 & 72,7 & 3 & 27,3 \\ 20 \text { a } 34 & 34 & 34,0 & 66 & 66,0 \\ 35 \text { a } 49 & 69 & 48,0 & 74 & 52,0 \\ 50 \text { a } 64 & 64 & 50,0 & 64 & 50,0 \\ 65 \text { a } 79 & 21 & 37,5 & 35 & 62,5 \\ \geq 80 & 3 & 37,5 & 5 & 62,5\end{array}$

\begin{tabular}{lrrrrrr}
\hline $\begin{array}{l}\text { Escolaridade } \\
\text { (anos de estudo) } \\
\text { nenhuma }\end{array}$ & 24 & 11,9 & 25 & 9,9 & $1,69(0,53-5,48)$ & $>0,05$ \\
1 a 3 & 34 & 16,8 & 31 & 12,3 & $1,48(0,4904,57)$ & $>0,05$ \\
4 a 7 & 65 & 32,0 & 94 & 37,3 & $2,35(0,85-6,63)$ & $>0,05$ \\
8 a 11 & 27 & 13,3 & 41 & 16,3 & $2,47(0,81-7,60)$ & $>0,05$ \\
$\geq 12$ & 13 & 6,4 & 8 & 3,2 & 1,0 (referência) & - \\
ignorada & 40 & 19,6 & 53 & 21 & - & \\
\hline
\end{tabular}

OR: odds ratio com intervalo de confiança a $95 \%$.

apresentou mais anos de estudo ( 8 a 11 anos). A população de oitenta anos ou mais, em sua maioria $(62,5 \%)$, não estudou nenhum ano. Segundo a Tabela 2, dos portadores de hanseníase que não tiveram nenhuma escolaridade, 11,8\% eram mulheres e 9,9\% eram homens. De 8 a 11 anos de estudos, os homens são responsáveis por 16,3\% dos casos e as mulheres por 13,3\%. Porém, com 12 anos e mais de estudo houve predomínio de mulheres $(6,4 \%)$, em detrimento dos homens $(3,2 \%)$.

Observou-se que no total dos anos, a classe multibacilar representou $85,3 \%$ e a paucibacilar $12,9 \%$ dos casos, sendo que houve uma associação significativa entre a classificação multibacilar e o sexo feminino com uma chance quatro vezes superior em relação aos homens (OR = 4,4 [IC95\% 2,22 - 9,05]; p < 0,05). A baciloscopia mostrou-se negativa na maioria dos casos ao longo dos anos (52,1\%). A baciloscopia positiva foi verificada em $34,1 \%$ dos casos. Não foi realizado o exame de baciloscopia em 20,7\% dos casos em 2003 e em 10,7\% dos casos de 2006 foram assinalados como ignorados ou em branco. Em todos os casos em $<1,15-19$ e 80 anos e mais ocorreu a hanseníase na forma multibacilar. Em todas as faixas etárias, a classificação de multibacilar prevaleceu em mais de $75 \%$ dos casos, exceto a de 5 a 9 anos, que houve uma porcentagem de $50 \%$ para multibacilar e paucibacilar.

Na faixa etária mais acometida pela hanseníase (35 a 49 anos), $31,4 \%$ dos casos apresentaram incapacidade de grau zero e $11,2 \%$ apresentaram incapacidade grau I. Na população de 15 a 19 anos, 18,2\% apresentou incapacidade grau I (Tabela 3). Trinta e três vírgula oito por cento das pessoas que tiveram a sua avaliação de incapacidade ignorada ou deixada em branco tiveram mais que 5 lesões. Das que possuíam grau zero de incapacidade, $29,7 \%$ tinham lesão única e 29,9\% tinham mais que cinco lesões. Os portadores de incapacidade grau I tiveram 
TABELA 3 - Distribuição percentual dos casos de hanseníase por faixa etária, segundo grau de incapacidade na alta - Uberaba/MG, 2000 e 2006.

\begin{tabular}{|c|c|c|c|c|c|c|}
\hline \multirow[b]{3}{*}{ Faixa etária $\mathrm{X}$ incapacidade de cura na alta(ano) } & \multicolumn{6}{|c|}{ Grau de incapacidade } \\
\hline & \multicolumn{2}{|c|}{ Grau Zero } & \multicolumn{2}{|c|}{ Grau I } & \multicolumn{2}{|c|}{ Grau II } \\
\hline & $\mathrm{n}^{\mathrm{o}}$ & $\%$ & $\mathrm{n}^{\mathrm{o}}$ & $\%$ & $\mathrm{n}^{\mathrm{o}}$ & $\%$ \\
\hline$<1$ & & - & & - & & - \\
\hline 5 a 9 & 2 & 50,0 & & - & & - \\
\hline 10 a 14 & 3 & 75,0 & & - & & - \\
\hline 15 a 19 & 4 & 36,4 & 2 & 18,2 & 1 & 9,1 \\
\hline 20 a 34 & 20 & 20,0 & 8 & 8,0 & 1 & 1,0 \\
\hline 35 a 49 & 45 & 31,5 & 16 & 11,2 & 6 & 4,2 \\
\hline 50 a 64 & 41 & 32,0 & 16 & 12,5 & 5 & 3,9 \\
\hline 65 a 79 & 13 & 23,2 & 14 & 25,0 & 3 & 5,4 \\
\hline$\geq 80$ & & - & 2 & 25,0 & 1 & 12,5 \\
\hline
\end{tabular}

a mesma porcentagem, 29,3\%, para nenhuma lesão e para mais que cinco lesões. Quarenta e um vírgula dois por cento dos acometidos por incapacidade de grau II não possuíam nenhuma lesão.

Houve um maior índice de pessoas acometidas com mais de cinco lesões entre aqueles que fizeram uso de PQT de 12 (32,3\%) e de $24(37,9 \%)$ doses. Trinta e três vírugula três por cento dos pacientes que fizeram tratamento com PQT de 24 doses tiveram sua avaliação, quanto a lesões, ignoradas ou deixadas em branco.

A maioria dos portadores de hanseníase estudou em média de 4 a 7 anos (34,9\%). A faixa de 20 a 34 anos foi a que apresentou mais anos de estudo (8 a 11 anos). A população de oitenta anos ou mais, em sua maioria (62,5\%), não estudou nenhum ano. Segundo a Tabela 2 , dos portadores de hanseníase que não tiveram nenhuma escolaridade, $11,8 \%$ eram mulheres e $9,9 \%$ eram homens. De 8 a 11 anos de estudos, os homens são responsáveis por $16,3 \%$ dos casos e as mulheres por $13,3 \%$. Porém, com 12 anos e mais de estudo houve predomínio de mulheres (6,4\%), em detrimento dos homens (3,2\%).

Apresentaram lesão única, 78,3\% de pessoas que fizeram uso de esquema poliquimioterápico de seis doses. Houve um maior índice de pessoas acometidas com mais de cinco lesões entre aqueles que fizeram uso de PQT de $12(32,3 \%)$ e de 24 doses (37,9\%). Trinta e três por cento dos pacientes que fizeram tratamento com $\mathrm{PQT}$ de 24 doses tiveram sua avaliação, quanto a lesões, ignoradas ou deixadas em branco.

\section{DISCUSSÃO}

Observou-se uma elevação dos casos de hanseníase, principalmente em 2004, com 93 casos, quase o dobro de 2002. Achados descritos por outros autores ${ }^{4,11,12}$ apontam que os índices de hanseníase atingiram seu pico em 2002. Infere-se que em 2004 a elevação dos casos foi decorrente da busca ativa feita pela Secretaria Municipal de Saúde na comunidade, com o intuito de alcançar o proposto pelo Plano de Eliminação da Hanseníase 20002005. Os administradores de saúde precisam conhecer o quadro epidemiológico de sua região e elencarem ações de acordo com as prioridades de sua população ${ }^{4}$. Além de definirem áreas de risco para que diretrizes epidemiológicas sejam implantadas especificamente para aqueles problemas ${ }^{13}$. Este fator também pode estar relacionado com a grande imigração de trabalhadores cortadores de cana, advindos do norte, nordeste e centro-oeste do país, cujas regiões revelaram índices de prevalência de hanseníase, em 2004, de 4,8 e 2,0 e 3,8/10.000 habitantes respectivamente ${ }^{9}$. As precárias condições de saúde, a falta de higiene, a falta de saneamento básico, ou seja, fatores sociais e condições socioeconômicas a que essa população está sujeita, corroboram para que ela seja propensa à hanseníase ${ }^{14,15}$. Apesar de o estudo ter como enfoque os residentes em Uberaba, nosso município é referência no serviço de saúde para municípios vizinhos que controlam este tipo de mão de obra. Consequentemente, o município absorve uma parcela dessa população que pode estar contaminada por hanseníase e expõe aos seus moradores o contato com indivíduos possivelmente de risco.

Das pessoas acometidas, $31,4 \%$ estavam entre a faixa etária de 34 a 49 anos, e ainda 53,4\% representam o somatório da faixa etária de 20 a 49 anos. Isso nos indica que a população economicamente ativa é a mais afetada pela hanseníase, o que pode prejudicar a economia do município, visto que essa faixa da população pode vir a desenvolver incapacidades, lesões, estados reacionais, afastar-se da atividade produtiva e gerar um custo social demasiado ${ }^{13,15}$. Associada a essa questão está o fato de a forma clínica mais comum em todos os afetados, ser a dimorfa $(69,1 \%)^{16}$, de grande poder de transmissão, seguida pela virchowiana $(17,1 \%)$, alto poder de transmissão e alto poder de incapacidade, o que está de acordo com Goulart cols ${ }^{17}$. O que nos faz inferir que os indivíduos economicamente produtivos estão comprometidos com a doença e são os grandes responsáveis pela transmissão no município, de modo que a faixa etária entre 20 e 49 anos representa 117.735 do total da população de Uberaba, ou seja, $46,7 \%$.

A classificação operacional da hanseníase revelou que a ocorrência de casos de multibacilares no município foi predominante, e teve uma relação diretamente proporcional com o aumento da idade, semelhante a estudos anteriores ${ }^{4,12,16-18}$ exceto nos menores de dez anos. Essa relação da forma clínica com a idade pode ser decorrente do longo período de incubação da doença, somado ao não diagnóstico precoce.

A frequência encontrada com relação à cor, zona de residência e sexo está de acordo com a literatura ${ }^{1,4,19,20}$. De todo período avaliado, os homens somam $55,4 \%$ dos casos, de um modo geral, podemos inferir que os homens são os maiores responsáveis pela transmissão da hanseníase, assim como descrito por Curto e Paschoal ${ }^{9}$. Ao se analisar as características entre os gêneros, apesar de a hanseníase ser mais prevalente em homens, no presente estudo, constatou-se uma chance superior a quatro vezes $(O R=4,4 ; p<0,05)$ de se encontrar a doença multibacilar entre as mulheres, fato também observado em outro estudo ${ }^{1}$. Alguns estudos mostram que a hanseníase afeta mais homens que mulheres ${ }^{1}$; no entanto, há exceções como em Governador Valadares, MG, em que 55,3\% dos acometidos são mulheres ${ }^{21}$ bem como no estudo de Gomes cols, realizado em Fortaleza, Ceará ${ }^{22}$. O sexo masculino prevaleceu em praticamente todos os anos avaliados, exceto em 2005 e 2006, em que as mulheres apresentaram um aumento de $1,2 \%$ e 3,6\% respectivamente, fato semelhante é descrito por Campos cols ${ }^{23}$, em estudo realizado em Sobral, $\mathrm{CE}^{23}$. Essa pequena elevação dos índices pode ser decorrente do aumento de mulheres infectadas, ou devido a uma maior identificação dessas portadoras, pelo fato de terem mais acesso ao serviço de saúde e serem mais preocupadas com a autoimagem do que os homens ${ }^{1,23}$.

A baciloscopia foi realizada em $392(86,2 \%)$ pessoas e 155 $(34,1 \%)$ delas apresentaram valores positivos, indicando um grande risco de transmissão da doença, conforme descrito por Hinrichsen $\operatorname{cols}^{1}$. Um fator agravante é a detecção de hanseníase em menores de 15 anos, que é entendido como um indicador da alta transmissão da 
doença ocasionado muitas vezes pelos familiares, principalmente na infância em que há uma maior exposição ao bacilo ${ }^{12,13}$. Neste estudo, encontrou-se nove casos de hanseníase em menores de 15 anos, seis deles eram dimorfos, o que significa que essas crianças estão sendo avaliadas tardiamente, já com a capacidade de transmissão da doença com possíveis incapacidades ${ }^{13}$. O que aumenta o número de disseminadores da doença, pelo fato dessa faixa populacional estar em contato com um grande número de outros jovens nas escolas, em torno de cinco horas, em muitos dias do ano. Deve-se ter uma avaliação ativa dessa faixa etária para detectar inicialmente a hanseníase, ainda na fase indeterminada, para tentar impedir reações da infecção tardia, bem como os preconceitos sociais e problemas psicológicos ${ }^{10,12,24}$.

Ao cruzarmos sexo e faixa etária percebemos que o sexo masculino prevalece em quase todas as faixas etárias, porém de 15 a 19 anos, o sexo feminino predominou com $73 \%$ dos casos. Nos anos de 2005 e 2006, o número de casos femininos ultrapassou o masculino. Essa elevação de casos femininos também é relatada por outros autores ${ }^{15,19,20}$. Hinrichsen $\operatorname{cols}^{17}$ encontraram em sua pesquisa, que o sexo masculino foi predominante em todas as faixas etárias, porém, de 18 a 34 anos os valores se igualaram. O modelo cultural sociológico pode ser um meio de explicar as diferenças da hanseníase, quando se compara o sexo ${ }^{14}$. Os valores elevados de 15 a 19 anos merecem atenção, visto que as incapacidades e deformações que a doença pode ocasionar gerariam grandes efeitos na auto-estima e na autoimagem dessas adolescentes.

$\mathrm{Na}$ avaliação da incapacidade de cura, os dados deste estudo revelaram que, $48,8 \%$ dos casos foram ignorados ou deixados em branco, o que dificulta a visualização da gravidade das consequências da hanseníase e demonstra que a qualidade do preenchimento das Fichas Individuais de Investigação (FII) deve ser melhorada. O grau zero foi o que prevaleceu em todas as faixas etárias, assim como é descrito no trabalho de Gomes $\operatorname{cols}^{16}$. Nos pacientes que fizeram tratamento com esquema PQT de 24 doses, 37,9\% apresentaram em média cinco lesões pelo corpo, esse dado pode significar uma reação negativa do organismo à droga do tratamento.

A questão da escolaridade, que é muito importante para se realizar a educação em saúde dessa população revelou que 34\% dos avaliados haviam estudado no máximo até a sexta série no ensino fundamental. $\mathrm{O}$ que pode dificultar a apreensão das orientações sobre o tratamento e cuidados necessários. Porém, acima de 12 anos de estudos houve predomínio do sexo feminino, o que poderia ser explicado pela procura espontânea aos serviços de saúde pelas mulheres com maior escolaridade.

Observou-se que 35,3\% dos diagnósticos foram feitos através da demanda espontânea, ou seja, a população que procura o serviço de saúde, também observado por Lana cols ${ }^{21}$. Os exames coletivos são responsáveis por apenas $0,4 \%$ dos diagnósticos e os exames de contato por $2,4 \%$, valores muito baixos, assim como observado em estudos de Opromolla cols, na Cidade de Fortaleza, CE4 . Estes dois últimos são tidos como os principais instrumentos de avaliação da hanseníase, de modo que auxiliam na detecção mais precisa e mais precoce dos casos, ou seja, contribuem para diminuir os casos de prevalência oculta e diminuir as incapacidades ${ }^{13,21}$. Estes dados sugerem que a busca ativa é pouco implementada nos serviços de saúde do município de Uberaba, revelando uma falha no tratamento e na aplicação das diretrizes do plano de Eliminação da Hanseníase 2000-2005 e também do novo Plano Nacional de Eliminação da
Hanseníase de 2006-2010. Cabe ressaltar a grande importância dessa atividade em crianças menores de 15 anos $^{7}$, pois, de acordo com os dados encontrados, nove crianças apresentaram hanseníase e seis delas do tipo dimorfa. Selvasekar cols ${ }^{12}$ discorrem sobre a eficácia e importância da busca ativa, pois colabora com a identificação inicial de hanseníase e também com o controle de regiões afetadas pela doença ${ }^{12}$.

O município é considerado pólo no serviço de saúde para 27 municípios vizinhos. O serviço de saúde está iniciando a descentralização da assistência aos portadores de hanseníase, o que colabora para um aumento da área de abrangência do serviço e fica mais próximo à população. A hanseníase no município parece caminhar para o alcance do índice proposto pela OMS $(1 / 10.000)$. Porém, este índice deve ser considerado com cautela se considerarmos que casos podem estar ocultos (prevalência oculta), visto que a maioria é de portadores da doença multibacilar, seguido pela virchowiana, ou seja, disseminadores da doença. A busca ativa é falha no município, o que acarreta dados subnotificados no Sistema de Informação e Agravo de Notificação (SINAN). Ela é uma ferramenta muito importante no combate à doença, pois identifica precocemente os casos na comunidade, evita um aumento do número de casos, identifica os acometidos que abandonaram o tratamento, bem como detecta a doença na fase inicial (indeterminada), contribuindo para que reduza a presença de incapacidades, exclusão social e estigma. Em um estudo feito em São Paulo, através da busca ativa eles identificaram um número de portadores cem vezes mais do que os números oficiais do Ministério da Saúde9. Entretanto, somente a busca ativa não resolverá o problema da hanseníase, ela ajuda com uma identificação mais completa dos casos, porém ainda se faz importante uma continuação das ações. São necessárias intervenções, como uma educação continuada com abordagem clara, simples e objetiva do tema, com adequação ao nível social da clientela; expansão da cobertura do atendimento, com auxílio das Estratégias de Saúde da Família, acarretando na capacitação desses profissionais atuantes nessa área, e a busca, tratamento e acompanhamento dos familiares dos portadores.

Outro fator de extrema relevância é o fato de a hanseníase ser tida como uma doença estigmatizadora. $\mathrm{O}$ pouco conhecimento que a população detém dificulta a aceitação, até mesmo dos próprios portadores, que abandonam ou se recusam a realizar o tratamento, além de não admitirem que possuem a doença. Logo, urge que o tema tenha uma abordagem ampla perante a população, que haja um planejamento de uma educação continuada com o intuito de informar, esclarecer e educar essa comunidade.

\section{CONFLITO DE INTERESSE}

Os autores declaram não haver nenhum tipo de conflito de interesse.

\section{REFERÊNCIAS}

1. Hinrichsen SL, Pinheiro MRS, Juca MB, Rolim H, Danda GJN, Danda DMR. Aspectos epidemiológicos da hanseníase na cidade de Recife, PE em 2002. Anais Bras Dermatol 2004; 79: 413-421.

2. Moreira TA. Panorama sobre a hanseníase: quadro atual e perspectivas. Hist Cienc Saude Manguinhos 2003; 10: 291-307.

3. Opromolla PA, Dalben I, Cardim M. Análise da distribuição espacial da hanseníase no Estado de São Paulo, 1991-2002. Rev Bras Epidemiol 2005; 8: 356-364. 
4. Opromolla PA, Dalben I, Cardim M. Análise geoestatística de casos de hanseníase. Rev Saúde Pública 2006; 40: 907-913.

5. Ministério da saúde. Casos de hanseníase caem $24,27 \%$ em dois anos. Portal da Saúde, 2006. Disponível em http://portal.saude.gov.br/portal/aplicacoes/ busca/buscar.cfm. Acesso em 17 maio de 2007.

6. Curto M, Paschoal VD. Uma década de acompanhamento dos portadores de hanseníase no ambulatório de dermatologia de um hospital-escola. Arq Cienc Saude 2005; 12: 183-195

7. Lana FCF, Amaral EP, Lanza FM, Lima PL, Carvalho ACN, Diniz LG. Hanseníase em menores de 15 anos no Vale do Jequitinhonha, Minas Gerais, Brasil. Rev Bras Enferm 2007; 60: 696-700.

8. Loureiro VB, Alessi SS, Maragno L, Margarido LC. Campanha voluntária em comunidades carentes para diagnóstico precoce da moléstia de Hansen - integração docente, discente e assistencial. Rev Med São Paulo 2006; 85: 5057.

9. Ministério da Saúde. Departamento de Informação e Informática do SUS. DATASUS. Informações de Saúde: Epidemiológicas e Morbidades, 2006. Disponível em: http://tabnet.datasus.gov.br/cgi/tabcgi.exe?hans/hanswMG. def. Acesso em 27 de maio de 2007.

10. Mahajan PM, Jogaikar DG, Mehta JM. Study of deformities in children with leprosy: an urban experience. Indian J Lepr 1995; 67: 405-409.

11. Noordeen SK. Elimination of leprosy as a public health problem: Progress and prospects. Bull World Health Organ 1995; 73: 1-6.

12. Selvasekar A, Geetha J, Nisha K, Manimozhi N, Jesudasan K, Rao PS. Childhood leprosy in endemic area. Lepr Rev 1999; 1: 21-27.

13. Lana FCF, Amaral EP, Franco MS, Lanza FM. Estimativa da prevalência oculta da hanseníase no Vale do Jequitinhonha: Minas Gerais. Rev Min Enferm 2004; 8: 295-300.

14. Helene LMF, Salum MJL. A reprodução social da hanseníase: um estudo do perfil de doentes com hanseníase no Município de São Paulo. Cad Saúde Pública 2002; 18: 101-113.

15. Araújo MG. Hanseníase no Brasil. Rev Soc Bras Med Trop 2003; 36: 373-382.

16. Gomes CCD, Pontes MAA, Gonçalves HS, Penna GO. Perfil clínicoepidemiológico dos pacientes diagnosticados com hanseníase em um centro de referência na região nordeste do Brasil. Anais Bras Dermatol 2005; 8 (supl 3): 283-288.

17. Goulart IMB, Arbex GL, Carneiro MH, Rodrigues MS, Gadia R. Efeitos adversos da poliquimioterapia em pacientes com hanseníase: um levantamento de cinco anos em um Centro de Saúde da Universidade Federal de Uberlândia. Rev Soc Bras Med Trop 2002; 35: 453-460.

18. Bechelli LM, Dominguez VM, Patwary KM. WHO Epidemiologic random sample surveys of leprosy in Northern Nigeria (Katsina), Cameroon and Thailand (Khon Kaen). Int J Lepr Other Mycobact Dis 1966; 34:223-243.

19. Aquino DMC, Caldas AJM, Silva AAM, Costa JML. Perfil dos pacientes com hanseníase em área hiperendêmica da Amazônia do Maranhão, Brasil. Rev Soc Bras Med Trop 2003; 36: 57-64.

20. Ferreira IN, Alvarez RRA. Hanseníase em menores de quinze anos no município de Paracatu, MG (1994 a 2001). Rev Bras Epidemiol 2005; 8: 41-49.

21. Lana FCF, Lanza FM, Velásquez-Melendez G, Branco AC, Teixeira S, Malaquias LCC. Distribuição da hanseníase segundo sexo no Município de Governador Valadares, Minas Gerais, Brasil. Hansenol Int 2003; 28: 131-137.

22. Figueiredo IA. Detecção da hanseníase em São Luís-Maranhão de 1993 a 1998 Dissertação de Mestrado, Universidade Federal do Maranhão, São Luís, MA, 2001 .

23. Campos SSL, Ramos AN, Kerr-Pontes LRS, Heukelbach J. Epidemiologia da hanseníase no Município de Sobral, Estado do Ceará-Brasil, no Período de 1997 a 2003. Hansenol Int 2005; 30: 167-175.

24. Lastoria JC, Putinatti MSMA. Utilização de busca ativa de hanseníase: relato de uma experiência de abordagem na detecção de casos novos. Hansenol Int 2004; 29: 6-11. 